\title{
ANÁLISIS DIDÁCTICO DE PRÁCTICAS MATEMÁTICAS INSTITUCIONALES DE DIVISIBILIDAD REALIZADO DURANTE UN CURSO DE FORMACIÓN DOCENTE
}

Mgter. ESPINOZA, Ricardo Fabián ${ }^{(*)}$

Palabras clave: Análisis didáctico, prácticas matemáticas institucionales, tipos de problemas de divisibilidad, configuraciones epistémicas, funciones semióticas.

Resumen: En este trabajo se describen los principales aspectos del análisis didáctico realizado en prácticas institucionales de Divisibilidad, en el ámbito de un curso de formación de docentes del Nivel Medio. Dicho análisis se elabora usando herramientas teóricas y metodológicas del Enfoque Ontosemiótico del conocimiento y la instrucción matemáticos. Mediante el mismo, queda al descubierto una gran variedad de tipos de problemas a los que responde la Divisibilidad en el Nivel Medio y, en una práctica institucional de resolución de un problema particular, se puede apreciar la trama de funciones semióticas necesarias de establecer.

Introducción: En este trabajo se describen los principales aspectos del análisis didáctico realizado en prácticas institucionales de Divisibilidad, en el ámbito de un curso de formación de docentes del Nivel
Medio, llevado a cabo en la Facultad de Ciencias Exactas y Naturales y Agrimensura de la Universidad Nacional del Nordeste, en el segundo cuatrimestre del año 2.016.

Dicha capacitación, de título "La Comprensión de la Divisibilidad en el ingreso a la Universidad", se desarrolló en el marco del proyecto de extensión que dirijo en esta Facultad, que tiene por denominación: Aprendiendo a enseñar Matemática en el Nivel Medio y Superior.

En la primera parte de esta presentación se exponen unos tipos de problemas a los que responde la Divisibilidad en el Nivel Medio, los cuales fueron elaborados en la capacitación desarrollada, a partir del análisis de documentos curriculares, libros de textos de uso frecuente en el Nivel Medio y libros de Matemática Superior. En la segunda parte, se exhibe un problema representante de un tipo de problemas (expuesto en la primera parte) y el análisis didáctico realizado sobre la práctica institucional de su resolución, para el que se emplean herramientas teóricas y metodológicas provenientes del Enfoque Ontológico y Semiótico del conocimiento y la instrucción matemáticos. 
El marco teórico adoptado confiere sustancial importancia a la reconstrucción de tipos de problemas a los que resuelve un determinado objeto matemático y al análisis didáctico institucional, realizado por el profesor sobre problemas o tipos de problemas, como referencia para el análisis de las prácticas (personales) de los estudiantes; trabajo que persigue el propósito de mejorar la enseñanza.

\section{Desarrollo de la propuesta}

Marco teórico: El Enfoque Ontosemiótico del conocimiento y la instrucción matemáticos es una teoría de la Didáctica de la Matemática iniciada por el grupo de investigación denominado "Teoría de la Educación Matemática”, de la Universidad de Granada, dirigido por Juan Díaz Godino, en la década de los 90.

Siguiendo a Godino, Fernández y Cajaraville (1996), se puede manifestar que los postulados del Enfoque Ontosemiótico del conocimiento y la instrucción matemáticos se relacionan principalmente con la Antropología, la Ontología y la Semiótica, pero también se articulan de manera coherente supuestos socioculturales y psicológicos. La Matemática se concibe como una actividad humana, intencionalmente orientada a la solución de cierto tipo de problemas, realizada en el seno de instituciones o comunidades de prácticas; actividad que está mediatizada y apoyada por los recursos lingüísticos y tecnológicos disponibles. De las prácticas o sistemas de prácticas realizadas para resolver problemas emergen dos categorías pri- marias de objetos matemáticos: institucionales (sociales, relativamente objetivas, del profesor) y personales (individuales o mentales, del alumno), por lo que se asume que la Matemática es, además de una actividad, un complejo de objetos culturales (institucionales), axiomática y deductivamente organizados.

Este enfoque confiere fundamental importancia a las nociones de significados institucionales y personales y concibe el significado de un objeto matemático, al que Godino (2002) define como "todo aquello que es indicado, señalado o nombrado cuando se construye, comunica o aprende matemática", en términos del sistema de prácticas ligadas a un tipo de problemas; es decir, concibe que el significado de un objeto matemático es el sistema de prácticas operativas y discursivas que una persona, institución o comunidad de prácticas realiza para resolver un cierto tipo de problemas en las que dicho objeto interviene (Godino et. al., 2009). En este ámbito se considera práctica matemática a toda actuación o manifestación (lingüística o no) realizada por alguien para resolver problemas matemáticos, comunicar a otros la solución, validar la solución y generalizarla a otros contextos y problemas. La noción de sistema de prácticas (operativas y discursivas), constituidas por las prácticas significativas para resolver un campo de problemas y compartidas en el seno de una institución, asume una concepción pragmática-antropológica de las matemáticas, tanto desde el punto de vista institucional como personal y la actividad de resolución de problemas se adopta como elemento 
central en la construcción del conocimiento matemático (D’Amore y Godino, 2007).

Para un análisis más fino de la actividad matemática, el Enfoque Ontosemiótico incluye seis tipos de objetos matemáticos primarios intervinientes o emergentes de sistemas de prácticas (D'Amore y Godino, 2007):

- Situaciones: problemas más o menos abiertos, aplicaciones intramatemáticas o extra-matemáticas, ejercicios;

- Lenguaje: términos, expresiones, notaciones, gráficos;

- Procedimientos: operaciones, algoritmos, técnicas de cálculo;

- Proposiciones: atributos de los objetos mencionados, que suelen darse como enunciados o proposiciones;

- Conceptos: definiciones o descripciones;

- Argumentaciones: que se usan para validar y explicar las proposiciones (sean deductivas o de otro tipo).

Estos objetos están relacionados entre sí por medio de una función semiótica, caracterizada, según D’Ámore y Godino, como una correspondencia (ya sea relación de dependencia o función) entre un antecedente (expresión, significante o representante) y un consecuente (contenido, significado, representado) que establece un sujeto, persona o institución de acuerdo con cierto criterio. Dicha correspondencia se establece entre dos objetos cuando uno de ellos se pone en lugar del otro o bien uno es usado por otro. Con la noción de función semiótica se evidencia el carácter netamente relacional de la actividad matemática y de los procesos que difunden el conocimiento matemático.

Los objetos matemáticos primarios están relacionados entre sí formando configuraciones, definidas como las redes de objetos intervinientes y emergentes de los sistemas de prácticas y las relaciones que se establecen entre los mismos al resolver un problema o un tipo de problemas. Estas configuraciones pueden ser epistémicas (redes de objetos institucionales) o cognitivas (redes de objetos personales) y persiguen la finalidad de analizar las prácticas matemáticas describiendo su complejidad ontosemiótica (Godino et al, 2005).

Tipos de problemas de Divisibilidad en el Nivel Medio

Determinar si un número entero, dado en su expresión decimal, factorial, factorial prima, en base al algoritmo de la división y en base a la propiedad distributiva es divisor, factor, divisible o múltiplo de otro número entero expresado en estas mismas formas.

- Hallar todos los divisores de un número entero (chico, grande, producto de números primos grandes y cuadrado perfecto).

- Determinar la cantidad de divisores naturales de un número entero.

- Determinar la cantidad de divisores enteros de un número entero.

- Identificar la cantidad de divisores de un número natural conociendo la cantidad de divisores de su duplo o cuadrado.

- Decidir si un número entero es primo, compuesto o cuadrado perfecto.

- Hallar un número entero con una cantidad dada de divisores. 
- Hallar el menor número entero positivo con una cantidad dada de divisores.

- Indicar la condición bajo la cual, en una división entera, el divisor de la misma es divisor del dividendo.

- Decidir qué tipo de fracción es $\mathrm{a} / \mathrm{b}$ cuando a es divisor de $\mathrm{b}$.

- Hallar un número entero, o más de uno, conociendo sus múltiplos enteros comprendidos entre dos números enteros positivos.

- Identificar una cifra de un número entero positivo, expresado en base 10, para que el mismo sea divisible por otro entero positivo.

- Determinar si la relación de orden que se establece entre dos números enteros, cuando uno de los números es divisor del otro, es la misma que la que se da entre dos números naturales.

- Decidir si existen dos números enteros distintos que tengan los mismos divisores y las condiciones bajo las que esto sucede.

- Encontrar el mínimo común múltiplo entre dos o más números enteros (iguales, coprimos, uno múltiplo del otro).

- Determinar por qué el mínimo común múltiplo entre dos o más números enteros es un entero positivo.

- Encontrar el máximo común divisor entre dos o más números enteros (iguales, coprimos, uno múltiplo del otro).

- Determinar la razón por la cual, para hallar el máximo común divisor de dos o más números enteros, basta listar los divisores positivos.
Análisis Ontosemiótico de una práctica de divisibilidad

En este apartado se expone un problema representante de un tipo de problema de Divisibilidad señalado en el apartado anterior.

Se explicitan los objetos matemáticos involucrados en la práctica institucional de resolución de tal problema y se analiza el carácter relacional de los mismos, en términos de funciones semióticas. Para llevar a cabo dicho análisis se elabora una configuración epistémica.

Este trabajo fue realizado en instancias de la capacitación mencionada.

Problema: Teniendo en cuenta que: $187=$ $11 \times 17$, ¿̇son correctas las siguientes afirmaciones?:

a) 17 es divisor de $11 \times 17$.

b) $11 \times 17+1870$ es múltiplo de 187 .

c) $11 \times 17+16$ es múltiplo de 187 .

En cada caso, fundamenta tu respuesta.

Se trata de un problema intra-matemático.

En el primer ítem, la tarea consiste en decidir si un número entero expresado en base 10 , es divisor de otro número entero expresado en su descomposición factorial prima. En el segundo apartado, la tarea consiste en determinar si un número entero, expresado en base a la propiedad distributiva, es múltiplo de otro número entero expresado en base 10. En el tercer apartado, la tarea consiste en determinar si un número entero, expresado en base al algoritmo de la división, es múltiplo de otro número entero escrito en versión decimal. 
La resolución de problemas de divisibilidad con números expresados según su descomposición factorial prima, en base a la propiedad distributiva y en base al algoritmo de la división, a pesar de ser solicitados desde los diseños curriculares, no son prácticas usuales en el nivel medio, por lo cual este trabajo se constituye en un aporte en ese sentido.

\section{Resolución}

En esta práctica de resolución institucional no se trata de desplegar todos los conocimientos que dispone el Profesor; se trata de ilustrar los procesos cognitivos que a priori se piensa que podría desarrollar un alumno cuya práctica es considerada adecuada o pertinente.

a) Se puede afirmar que 17 es divisor de $11 \mathrm{x} 17$, pues es un factor de la descomposición factorial prima de 11x17. Esto es así, pues, teniendo en cuenta la definición de divisor (concepto), podemos decir que 17 es divisor de $11 \times 17$, pues existe un número entero, el 11 , que multiplicado por 17 da por resultado $11 \times 17$.

b) E1 número $11 \times 17+1870$ está expresado en base a la propiedad distributiva, teniendo en cuenta que, como $11 \times 17$ = 187 y $1870=187 \times 10$, en función de la propiedad distributiva del producto respecto de la suma de números enteros, al número en cuestión $(11 \times 17+1870)$ selo puede escribir así: $187+187 \times 10$. Ahora bien, $187+187 \times 10=187 x(1+10)=187 \times 11$ (concepto y procedimiento),expresión que nos dice que el número dado en la consigna $11 \times 17+1870$, que también puede escribirse como $187+187 \times 10$, es múltiplo de
187, pues 187 es divisor del mismo, ya que, teniendo en cuenta la definición de divisor, el número de interés puede escribirse como 187x11 (concepto y argumento).

Entonces, cuando un número entero puede descomponerse en la suma de $n$ productos con un factor común entero, es decir, puede escribirse en base a la propiedad distributiva, dicho número es múltiplo de ese factor común (propiedad).

c) El número 11x17+16 está expresado en base al algoritmo de la división entera; el dividendo es $203(11 \times 17+16)$, el divisor es 17 , el cociente, 11 y el resto, 16 .

El primer múltiplo positivo de 187 es el mismo 187 y el múltiplo consecutivo es: 187+187 = 374 (procedimiento). El número $11 \times 17+16$ = 203, está comprendido entre estos dos múltiplos consecutivos de 187:

$187<203<374$, razón por la cual podemos afirmar que $11 \times 17+16$ no es múltiplo de 187, dado que, entre dos múltiplos consecutivos de un número entero, no existe otro múltiplo (argumento).

\section{Análisis del problema}

La configuración epistémica del problema resulta ser la descripta en el cuadro de la página siguiente.

\section{Red de funciones semióticas (FS) "in-} mediatas" presentes en la configuración epistémica:

FS1: Entre el problema y el procedimiento de observar si un número $\mathrm{p}$ es un número primo de la descomposición factorial de un número entero $\mathrm{n}$, para decidir que $\mathrm{p}$ es divisor de $\mathrm{n}$. 


\begin{tabular}{|c|c|c|}
\hline \multirow{6}{*}{ 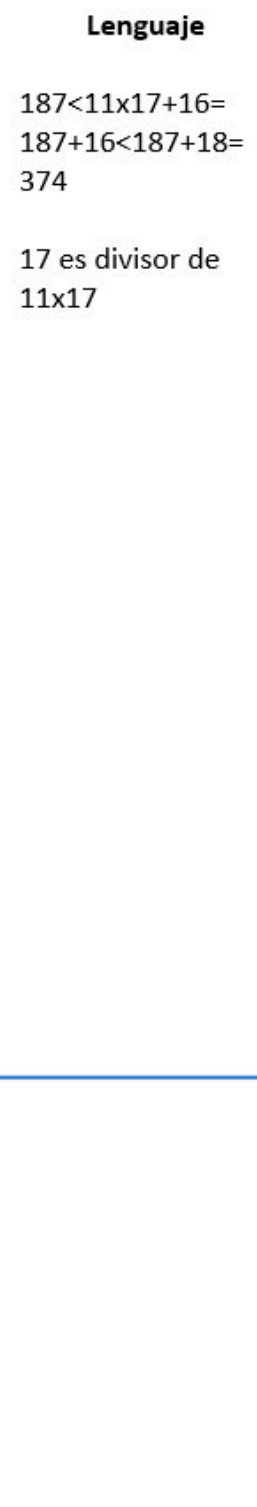 } & \multicolumn{2}{|c|}{$\begin{array}{l}\text { Situación Problemática } \\
\text { Situación intramatemática que implica determinar si un número entero } \\
\text { escrito en base 10, en función de la propiedad distributiva o el algoritmo de la } \\
\text { división entera, es múltiplo o divisor de otro número entero expresado en } \\
\text { algunas de estas mismas versiones. }\end{array}$} \\
\hline & & \\
\hline & \multirow{3}{*}{ 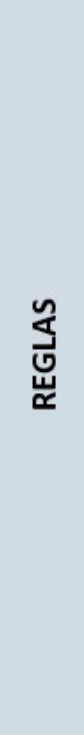 } & Definición de divisor. Conceptos \\
\hline & & $\begin{array}{l}\text { Propiedades } \\
\text { Cuando un número entero puede descomponerse en la suma de n } \\
\text { productos con un factor común entero, es decir, puede escribirse } \\
\text { en base a la propiedad distributiva, dicho número es múltiplo de } \\
\text { ese factor común. } \\
\text { Propiedad distributiva del producto respecto de la suma de } \\
\text { números enteros. }\end{array}$ \\
\hline & & $\begin{array}{l}\qquad \text { Procedimientos } \\
\text { Observar la descomposición factorial prima de un número entero. } \\
\text { Cualquier factor primo de tal descomposición, es divisor de dicho } \\
\text { número. } \\
\text { Hallar múltiplos consecutivos. } \\
\text { Escribir un número entero en base a la propiedad distributiva del } \\
\text { producto respecto de la suma. }\end{array}$ \\
\hline & $\begin{array}{r}-17 \\
-11 x \\
\text { es } \\
\text { la d } \\
- \text { Con } \\
\text { (el } \\
\text { ya } \\
\text { otrc }\end{array}$ & $\begin{array}{l}\text { Argumentos } \\
\text { divisor de } 11 \times 17 \text {, pues lo justifica la definición de divisor. } \\
+1870=187+187 \times 10=187 \times(1+10)=187 \times 11 \text {, por lo que } 11 \times 17+1870 \\
\text { Itiplo de } 187 \text {, pues } 187 \text { es divisor de } 11 \times 17+1870 \text {, teniendo en cuenta } \\
\text { nición de divisor. } \\
11 \times 17+16 \text { está comprendido entre dos múltiplos consecutivos de } 187 \\
7 \text { y el } 187+187) \text {, se puede afirmar que } 11 \times 17+16 \text { no es múltiplo de } 187 \text {, } \\
\text { e, entre dos múltiplos consecutivos de un número entero, no existe } \\
\text { últtiplo. }\end{array}$ \\
\hline
\end{tabular}

FS2: Entre el procedimiento que consiste en observar si un número $\mathrm{p}$ es un primo de la descomposición factorial de un número n para decidir que es su divisor y el concepto dado por la definición de divisor.

FS3: Entre el problema y el procedimiento que consiste en expresar a un número entero en base a la propiedad distri- butiva, dejando explícito el factor común, para decidir que el número en cuestión es múltiplo de ese factor común.

FS4: Entre el procedimiento que conlleva escribir un número entero en base a la propiedad distributiva, dejando explícito el factor común, y el concepto dado por la definición de divisor. 
FS5: Entre el procedimiento de escribir un número en base a la propiedad distributiva, dejando a la vista el factor común, y la propiedad que dice que cuando un número entero puede descomponerse en la suma de $\mathrm{n}$ productos con un factor común entero, es decir, puede escribirse en base a la propiedad distributiva, dicho número es múltiplo de ese factor común.

FS6: Entre el problema y el procedimiento de acotar el número $11 \times 17+16$ entre dos múltiplos consecutivos de 187.

FS7: Entre el procedimiento de acotar el número $11 \times 17+16$ entre dos múltiplos consecutivos de 187, y el argumento que explica que aquel número no es múltiplo de 187, ya que, entre dos múltiplos consecutivos de un número entero, no existe otro múltiplo.

\section{Conclusiones}

En el trabajo didáctico realizado con los docentes asistentes al curso mencionado se reconstruye una gran variedad de tipos de problemas a los que responde la Divisibilidad en el Nivel Medio.

Asimismo, se analiza y reflexiona sobre supuestos teóricos y metodológicos a fin de realizar análisis didácticos referen- ciales para el análisis de las prácticas de los estudiantes.

Estos trabajos permiten apartar a los profesores del "sentido común" con el que suelen orientar sus prácticas didácticas, acercándolos a la comprensión y uso de teorías didácticas.

Los destinatarios disponen ya de una amplia gama de problemas los que, propuestos en clases, podrían hacer emerger una gran variedad de relaciones conceptuales entre objetos matemáticos involucrados en las prácticas matemáticas de los estudiantes.

Además, cuentan también con buenas herramientas de análisis didáctico de prácticas institucionales, lo que sin duda se constituye en una importante referencia para analizar las prácticas matemáticas de los estudiantes, tareas éstas que le atribuyen mejores condiciones para elaborar propuestas de enseñanza pertinentes. En el análisis ontosemiótico del problema de divisibilidad (expuesto al final de este trabajo), los asistentes al curso no sólo pudieron dejar explícitos los objetos matemáticos involucrados en una práctica matemática de su resolución, sino también una importante red de relaciones conceptuales entre esos objetos. 


\section{BIBLIOGRAFÍA}

D'Amore, B. y Godino, J. (2007). E1 Enfoque ontosemiótico como un desarrollo de la teoría antropológica en didáctica de la matemática. Relime, 10(2), 191-218. Fernández, T.; Cajaraville, J. y Godino, J. (2007). Configuraciones epistémicas y cognitivas en tareas de visualización y razonamiento espacial. En M. Camacho; P. Flores y M. P. Bolea (Eds.). Investigación en educación matemática, pp. 189-198. Disponible en INTERNET: http://funes.uniandes.edu.co/1252/1/Fernandez2008Configuraciones_SEIEM_189. pdf Godino, J. (2003). Teoría de las funciones se- mióticas. Un enfoque ontológico semiótico de la cognición e instrucción matemática. Granada: Departamento de Didáctica de la Matemática de la Universidad de Granada. Godino, J.; Font, V.; Contreras, A. y Wilhelmi, M. (2005). Articulación de marcos teóricos en didáctica de las matemáticas. Disponible en INTERNET: http:// www4.ujaen.es/ aestepa/TAD/Comunicaciones/Godino_y_cols_Articulacion.pdf Godino, J.; Font, V.; Wilhelmi, M. y Arreche, M. (2009). ¿Alguien sabe que es el número? Revista Iberoamericana de Educación Matemática, 19, 34-46. 